\title{
The GRANIT project: Status and Perspectives
}

\author{
Stephan Baeßler, ${ }^{a}$ Alexei Gagarski, ${ }^{b}$ Ludmilla Grigorieva, ${ }^{b}$ Michael Kreuz, ${ }^{d}$ Fabrice \\ Naraghi, ${ }^{c}$ Valery Nesvizhevsky, ${ }^{d}$ Guillaume Pignol, ${ }^{c}$ Konstantin Protassov, ${ }^{c}$ \\ Dominique Rebreyend*, ${ }^{c}$ Francis Vezzu, ${ }^{c}$ Alexei Voronin ${ }^{e}$ \\ ${ }^{a}$ University of Virginia, Charlottesville, VA-22904, USA \\ and Oak Ridge National Laboratory, Oak Ridge, TN-37831, USA \\ ${ }^{b}$ PNPI, Orlova Roscha, Gatchina, Leningrad Reg. 188350, Russia \\ ${ }^{c}$ LPSC/UJF-IN2P3-INPG, 53 rue des Martyrs, Grenoble F-38026, France \\ ${ }^{d}$ ILL, 6 rue Jules Horowitz, Grenoble F-38042, France \\ ${ }^{e}$ Lebedev Institute, 53 Leninsky Pr., Moscow 119991, Russia \\ E-mail: baessler@virginia.edu, gagarski@pnpi.spb.ru, \\ l_grigoryeva@mail.ru, kreuz@ill.eu, naraghi@lpsc.in2p3.fr, \\ rebreyendelpsc.in2p3.fr,nesvizhevskyeill.eu, \\ pignolelpsc.in2p3.fr,protasovelpsc.in2p3.fr, \\ vezzu@lpsc.in2p3.fr,dr.a.voronin@gmail.com
}

\begin{abstract}
The GRANIT project is the follow-up of the pioneering experiments that first observed the quantum states of neutrons trapped in the earth's gravitational field at the Institute Laue Langevin (ILL). Due to the weakness of the gravitational force, these quantum states exhibit most unusual properties: peV energies and spatial extensions of order $10 \mu \mathrm{m}$. Whereas the first series of observations aimed at measuring the properties of the wave functions, the GRANIT experiment will induce resonant transitions between states thus accessing to spectroscopic measurements. After a brief reminder of achieved results, the principle and the status of the experiment, presently under commissioning at the ILL, will be given. In the second part, we will discuss the potential of GRANIT to search for new physics, in particular to a modified Newton law in the micrometer range.
\end{abstract}

The 2011 Europhysics Conference on High Energy Physics-HEP 2011,

July 21-27, 2011

Grenoble, Rhône-Alpes France

\footnotetext{
* Speaker.
} 


\section{The neutronic quantum bouncer}

Neutrons bouncing off a horizontal mirror under the sole gravitational attraction of the earth may sound unexpected or even impossible for a high energy physicist. Indeed, neutrons being neutral objects of extremely small dimension, they are known to be very penetrating particles. Moreover, gravity is by far the weakest of all forces and is usually totally negligible in experiments dealing with microscopic bodies. This very phenomenon has nevertheless been observed about 10 years ago in a pioneering experiment at the Institut Laue Langevin (ILL) [1], thanks to the use of very low energy neutrons. These neutrons, known as Ultra-Cold-Neutrons (UCN), have velocities of a few $\mathrm{m} / \mathrm{s}$, equivalent to energies in the $100 \mathrm{neV}$ range, and have the extraordinary property of being reflected at any incidence angle by some materials. As a consequence, they can be stored in containers and are the privileged tools to perform particle physics experiments at low energy, most notably measurements of the neutron lifetime and of its electric dipole moment.

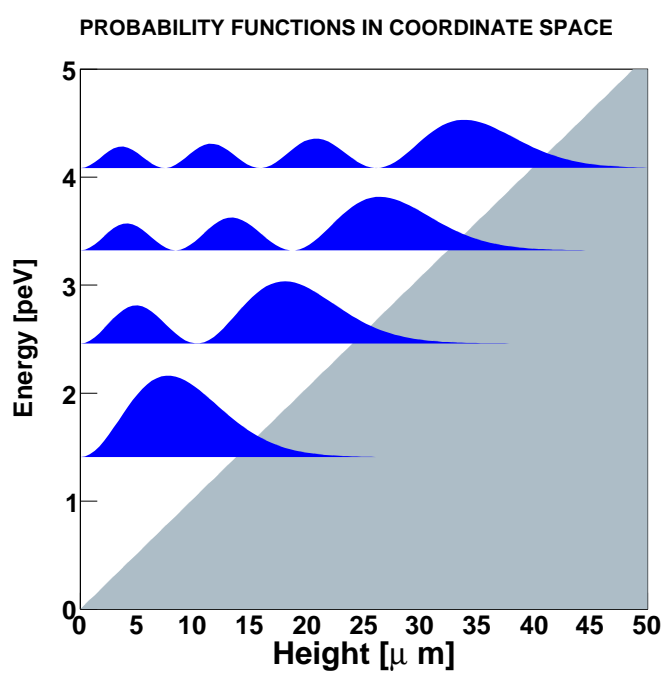

Figure 1: Energies and density probability functions of the first 4 quantum states.

In the language of quantum mechanics, neutrons are trapped in a potential well - formed by a nearly infinite wall due to the repulsion of the mirror ${ }^{1}$ on the one hand and the usual linear $m g z$ attractive potential of the earth on the other hand - and their energy is therefore discretized. Hence the name neutronic quantum bouncer.

Fig. 1 shows the energy and the probability density function of the first 4 quantum states. Because of the weakness of the gravitational interaction, these states have outstanding properties: energies are in the peV $-10^{-12} \mathrm{eV} !-$ range and the typical size of the wave-functions is of order $10 \mu \mathrm{m}$, i.e of macroscopic extension.

The very first experiment measured the flux of UCN after a slit of varying height, whose upper half was an absorber. In agreement with the vertical extension of the ground state, UCN could be detected after the slit only when its dimension would be larger than about $10 \mu \mathrm{m}$. Other

\footnotetext{
${ }^{1}$ Energy of the quantum levels of interest is $\sim 10^{-12} \mathrm{eV}$ to be compared to the $90 \mathrm{neV}$ repulsive (Fermi) potential of the quartz mirror.
} 
measurements were also performed but all based on the neutron vertical distribution i.e. testing only the wave-function of this system.

This series of pioneering experiments opened up a new field of research, giving one of the very few possibilities to study gravitation in the context of quantum mechanics [2, 3]. Numerous ideas have been proposed to exploit the high sensitivity of this system, in particular the possibility to constrain a short range fifth interaction. However, the precision reached on the parameters of this system, and correspondingly the sensitivity to new physics, was limited to a few percent and did not allow to improve the current limits $[4,5]$.

\section{The GRANIT project}

GRANIT aims at inducing transitions between the quantum states of the neutronic quantum bouncer in order to measure the transition energies. By doing so, we will access to the high precision of spectroscopic measurements and will increase our sensitivity to perturbations by orders of magnitude.

A scheme of the planned initial set-up is displayed in Fig.2. From left to right, the UCN will first be prepared in the third state by a step of appropriate height. They will then bounce between a mirror and a wire system whose function is to induce a Raby-like transition to the ground state. The wires are fed by steady currents and produce a spatially periodic magnetic field gradient. In their rest frame, neutrons will feel an oscillating gradient with a frequency depending on their velocity. Only neutrons of a given velocity will be at resonance, i.e. will see a gradient frequency equal to the $1 \rightarrow 3$ transition frequency, and will then undergo a full transition to the ground state. At the end of the mirror, a filter will select only UCN in the ground state. The surviving neutrons will then start a free fall until they reach a position-sensitive detector. By measuring the height of the free fall, one will be able to calculate the horizontal velocity and in fine to access to the $1 \rightarrow 3$ transition frequency.

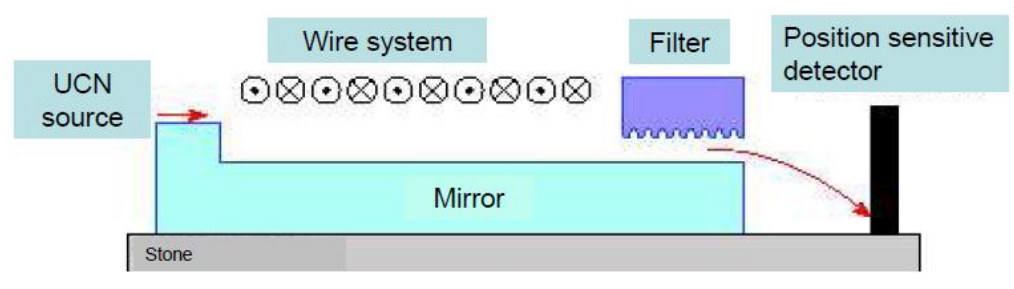

Figure 2: Principle of the set-up to measure resonant transitions induced by an oscillating magnetic field gradient in the flow-through mode.

It should be noted that the gravity-resonance-spectroscopy has been realized by Abele and collaborators [6]. Instead of using magnetic gradients, they used mechanical oscillations of the underlying mirror to induce the transitions.

Most of the equipment is now in place at level C of the ILL. Commissioning has already started and we expect to start real data taking next Spring. A more detailed description of the apparatus can be found in [7]. 


\section{Constraint on Chameleon models}

In a recent work [8], the potential detection of chameleons with the neutronic quantum bouncer has been explored. Chameleons are scalar fields of the quintessence type and are among the few serious candidates to explain the accelerated expansion of the Universe.

What makes these scalar fields of particular interest is that they couple to matter, hence acquiring the so-called chameleon mechanism, by which they could have evaded long range fifth force searches or equivalence principle tests. Because of the coupling to matter, the field gets trapped inside matter, screening its existence to the outside world. In [8], it has been shown that the existence of a chameleon field would produce an extra attractive term above the mirror and would lead to a modified gravity potential:

$$
\Phi(z)=m g z+\beta \frac{m}{M_{\mathrm{Pl}}} \phi(z) .
$$

with $\phi(z)$ the chameleon field, $m$ the mass of the neutron, $M_{\mathrm{Pl}}$ the Planck mass and $\beta$ the coupling constant. This extra term would lead to two potentially observable effects: the shrinking of the wave functions of the stationary states and the shifting of the energy levels. Fig. 3 shows the resulting exclusion plot.

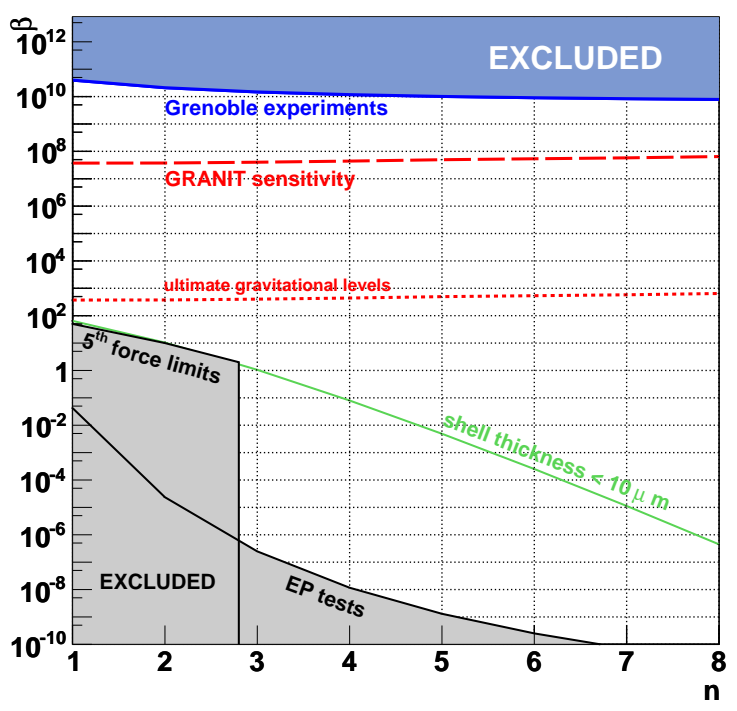

Figure 3: The chameleon exclusion plot. We find that above the bottom green line the chameleon field is independent of the coupling, above the top blue line chameleons produce a quantum state with a size of 2 microns and finally above the red dashed line the chameleons shift the $3 \rightarrow 1$ resonance by more than $0.01 \mathrm{peV}$. We have also drawn the ultimate sensitivity limit at the $10^{-7} \mathrm{peV}$ level.

Macroscopic experiments (Equivalence Principle tests and 5th force limits) exclude the small $\beta$ region. This is due to the screening effect: when $\beta$ increases, the chameleon thin shell at the surface of the test bodies shrinks and there is no increase in the force. For the neutronic quantum bouncer, the situation is different. Neutrons have no thin shell effect and, as seen in equation 3.1, the potential felt by the neutron is linear in $\beta$. The very large $\beta$ region is already excluded by the 
first series of experiments. For such large coupling, an additional bound state near the surface of the mirror would appear and would have been detected. In the first stage of the GRANIT experiment, the energy difference $E_{3}-E_{1}$ is expected to be measured with an accuracy of $0.01 \mathrm{peV}$ which sets the sensitivity to a chameleon induced shift (dashed line in fig. 3). GRANIT will be therefore able to set competitive constraints in the large coupling region of the chameleon models parameter space.

In summary, GRANIT is a second generation experiment aiming at investigating the properties of the neutron quantum bouncer, using the high precision of spectroscopic measurements. We expect to improve the precision of the first series of measurememts - thereby our sensitivity to new physics - by at least an order of magnitude within the next 3 years.

\section{References}

[1] V. V. Nesvizhevsky et al., Nature 415, 297 (2002).

[2] V. V. Nesvizhevsky and K. V. Protasov, "Quantum States of Neutrons in the Earth's Gravitational Field: State of the Art, Applications, Perspectives," in Trends in Quantum Gravity Research, edited by David C. Moore (Nova Science Publishers Inc., New York, 2006), p. 65.

[3] V. V. Nesvizhevsky, Physics-Uspekhi 53 (7): 645-675 (2010).

[4] V. V. Nesvizhevsky and K. V. Protasov, Class. Quant. Grav. 21, 4557 (2004).

[5] S. Baeßler, J. Phys. G: Nucl. Part. Phys. 36, 104005 (2009).

[6] T. Jenke, P. Geltenbort, H. Lemmel and H. Abele, Nature Physics 7, 468 (2011).

[7] M. Kreuz et al., Nucl. Instr. and Meth. A611, 326 (2009).

[8] P. Brax and G. Pignol, Phys. Rev. Lett. 107, 111301 (2011). 